\title{
¿Cómo elaborar el marco teórico de una tesis?
}

\author{
María Miroslava Olivarec-Bonilla* \\ Servicio de Urgencias, Hospital Regional con UMF N. ${ }^{\circ} 1$ Ignacio García Téllez, Instituto Mexicano del Seguro Social, Cuernavaca, Mor., México
}

\begin{abstract}
Resumen
El marco teórico es parte medular en los trabajos de investigación. Es el soporte y base de toda la investigación y cuenta con una estructura que guía al investigador, le ayuda a plantear preguntas de investigación y mantenerse enfocado, lo actualiza en el tema y documenta la necesidad de realizar el trabajo, le proporciona las bases de cómo habrá de realizarse el estudio, evita errores y el plagio, además de dar referencia para interpretar los resultados.
\end{abstract}

Palabras clave: Metodología. Investigación. Marco teórico.

\section{How to elaborate the theoretical framework of a thesis?}

\begin{abstract}
The theoretical framework is a central part of the research work. It is the support and basis of all research, has a structure that guides the researcher, helps to raise research questions and keeps the focus, updates on the subject and in the documents needed to carry out the work, it provides with the bases of how the study will be carried out, avoids errors and plagiarism, as well as giving reference to interpret the results.
\end{abstract}

Key words: Methodology. Research. Theoretical framework.

\section{Introducción}

Un trabajo de tesis consta de varios apartados: título, marco teórico, justificación, planteamiento, la pregunta de investigación, objetivos, hipótesis, metodología (tipo de estudio, población y muestra, procedimientos, variables e indicadores, técnicas de análisis y preceptos éticos, aprobación del comité), recursos, análisis y presentación de datos, cronograma y referencias bibliográficas.

Cuando seleccionamos el tema de estudio y planteamos la pregunta de investigación, es tiempo de construir el marco teórico. El marco teórico, también conocido como marco referencial, es la exposición y análisis de las teorías y antecedentes que sirven como fundamento para explicar los resultados de la investigación?

Se conocen tres marcos referenciales: teórico, conceptual y de antecedentes. El marco referencial teórico es un resumen de las teorías del tema. El marco referencial conceptual es un resumen de conceptos, la relación entre estos y la evolución (Ejemplo: sepsis 1-sepsis 3). Un concepto es una abstracción de la realidad, en una investigación estos conceptos deben ser los más

\section{Correspondencia:}

*María Miroslava Olivarec-Bonilla

E-mail: dra.miros@ hotmail.com
Fecha de recepción: 23-07-2019

Fecha de aceptación: 23-07-2019 DOI: 10.24875/REIE. 19000050
Disponible en internet: 06-11-2019 Rev Educ Investig Emer. 2019;1(3):119-123 www.medicinadeemergencias.com

2604-6520 @ 2019 Sociedad Mexicana de Medicina de Emergencias, AC. Publicado por Permanyer México SA de CV. Este es un artículo open access bajo la licencia CC BY-NC-ND (http://creativecommons.org/licenses/by-nc-nd/4.0/). 
precisos y evitar la ambigüedad; definir las variables de estudio conceptualmente y operacionalmente nos ayudará a clasificar y ordenar la información, por ejemplo: el infarto de miocardio es una necrosis del músculo cardiaco (definición conceptual), los criterios diagnósticos son el dolor anginoso, cambios en el electrocardiograma y elevación de marcadores cardiacos (definición operacional clara y medible). Para hacer investigación tenemos que tener claros y precisos los conceptos y el lenguaje acerca del tema. Finalmente el marco referencial de antecedentes es el resultado de investigaciones similares a la que pretendemos realizar y son los resultados de dichos estudios con los que vamos a comparar nuestros resultados ${ }^{3-5}$ (Tablas 1 y 2).

Para no perdernos en el proceso y que la lectura sea tediosa y sin resultados, vamos a llevar a cabo los siguientes pasos.

\section{Revisión de la literatura e identificación de las fuentes de información}

Nos referimos a la consulta en bases de datos y fuentes documentadas.

Estas son todos los recursos que contienen datos formales, informales, escritos, orales o multimedia. Se dividen en dos tipos: primarias y secundarias ${ }^{6}$.

Las fuentes primarias son aquellas diseñadas ex professo para recabar la información necesaria para el estudio, es información obtenida de primera mano, de estrategias de recolección mediante cuestionarios, mediciones intencionales, pruebas de laboratorios o imagen, entre otras ${ }^{7}$. Algunos autores consideran fuentes primarias aquellos trabajos que han sido publicados por primera vez, que no han sido evaluados e interpretados por nadie, como los artículos originales en revistas académicas, y bases de datos gubernamentales, como la del Instituto Nacional de Estadística y Geografía ${ }^{8}$.

Las fuentes secundarias son datos obtenidos de trabajos con propósitos distintos al estudio bajo conducción. Una fuente muy útil se obtiene a partir de las estadísticas oficiales de mortalidad basadas en el certificado médico de defunción. Las fuentes secundarias contienen información primaria sintetizada y reorganizada por y para otros investigadores, son compilaciones, diccionarios, enciclopedias especializadas, resúmenes publicados en revistas en un área del conocimiento. La revisión de la literatura servirá para actualizarse en el tema, encontrar puntos de vista posiblemente diferentes al propio y motivar el propio trabajo. Las fuentes secundaria son como «colegas" que hablan el mismo tema acerca de cómo lo escribió, qué
Tabla 1. Atributos del marco teórico

El marco teórico tiene atributos que ayudan a desarrollar tu trabajo de investigación:

1 Define y aísla el problema a investigar (identificando variables de estudio)

2 Revisa las teorías y antecedentes (estudios similares al que se plantea realizar)

3 Explora la metodología (diseños de estudio, muestras, tipo de participantes)

$4 \quad$ Instrumentos de medición utilizados

5 Orienta y evita errores al analizar cómo se han realizado estudios similares

6 Orienta sobre la hipótesis de trabajo

7 Evidencia otras preguntas de investigación que no han sido planteadas

Adaptada de Munch, et al., 2009 .

Tabla 2. Preguntas que debería responder, como mínimo, un marco teórico de un trabajo de tesis

\section{Un marco teórico responde las siguientes preguntas:}

$1 \quad$ ¿Existen información y estudios en relación con la pregunta de investigación?

2 ¿Existen estudio recientes (últimos 5 años)? ¿Qué dicen?

3 Los estudios disponibles, ¿responden a la pregunta de investigación?

4 Los resultados de los estudios, ¿son aplicables en nuestra población?

5 ¿Qué enfoque al problema le han dado en estudios similares al que pretendemos realizar?

6 ¿Qué hipótesis se han generado?

7 ¿Qué metodología se ha utilizado?

8 ¿Qué instrumentos de recolección o medición se han usado?

Adaptada de Munch, et al., 2009?.

analizó, qué estilo utilizó, y cómo desarrolló su escrito e investigación $n^{5,7,8}$.

Bases de datos electrónicos que podemos consultar: Medline (NLM), Index Medicus y LILACS EMBASE.

\section{Establecer una estrategia de búsqueda}

Lo primero es identificar los conceptos relacionados con la pregunta de investigación. Un ejemplo sería la formulación de la pregunta de investigación con la 
estrategia PICO: Patient/Problem, Intervention, Comparation, Outcome (población, intervención, comparación y resultados); paciente $=$ pacientes con infarto de miocardio, intervención = trombólisis, comparación = tiempo, resultados = causas de retraso. La pregunta sería ¿cuáles son las causas de retraso de trombólisis en paciente con infarto de miocardio?. Ya tenemos identificados al menos tres conceptos. Es necesario de igual forma identificar las palabras clave (key words), por ejemplo, hace algún tiempo al elaborar el marco teórico sobre el tiempo puerta-aguja y causas de retraso en trombólisis en pacientes con infarto de miocardio, al utilizar la palabra tiempo puerta-aguja en el buscador, no encontré ni un solo artículo, en cambio usando las palabras clave (key words) infarto de miocardio, retraso y trombólisis, y en idioma inglés myocardial infarction, therapy y delay encontré varios artículos ${ }^{9}$.

En una búsqueda los descriptores (palabras o frases controladas) nos ayudan a obtener resultados más precisos, reduciendo el número de trabajos irrelevantes a los intereses del autor; la terminología MeSh (Medical Subject Heading) es el vocabulario controlado que emplean Medline y otras bases de datos biomédicas para procesar la información que se introduce en cada una de ellas; existe una traducción al español que se conoce como «descriptores en ciencias de la salud» (DeCS). Ambos tipos de descriptores están disponibles en internet. Recomiendo revisar las palabras clave que utilizaron en estudios similares al que pretende realizar ${ }^{10}$.

\section{Criterios de selección}

Una vez que ya tenemos una serie de documentos revisaremos en el resumen si cubre las necesidades de nuestra investigación, tipo de población, estudios en adultos o niños, grupo de edad, región geográfica en México, América Latina, tipo de estudios epidemiológicos o analíticos, sobre diagnósticos (prueba diagnóstica) y tratamientos (ensayos clínicos) ${ }^{11}$.

\section{Organización de la información}

Se recomienda la elaboración de fichas bibliográficas. Las fichas bibliográficas deben de tener los siguientes elementos: título, referencia, texto o un código de identificación; pueden ser de libros, artículos de revistas, páginas web, tesis y otras fuentes información. Se clasifican en fichas textuales y el contenido es tal cual la trascripción de la idea del autor (este se anota entre comillas), de resumen se anota de forma sintetizada y con palabras propias los conceptos más
Tabla 3. Ejemplo de ficha bibliográfica

\section{Ficha bibliográfica de parafraseo}

Asimos AW, Johnson AM, Rosamond WD, et al. A multicenter evaluation of the ABCD2 score's accuracy for predicting early ischemic stroke in admitted patients with transient ischemic attack. Ann Emerg Med. 2010;55:201-10. (Citación estilo Vancouver.)

La escala $A B C D 2$ discrimina bien entre eventos no cerebrovasculares y los que sí lo son; por ejemplo, los factores de la escala ABCD2 "debilidad unilateral" y "alteración del lenguaje» son más prevalentes en los pacientes con ataque isquémico transitorio (AIT), comparados con eventos no cerebrovasculares.

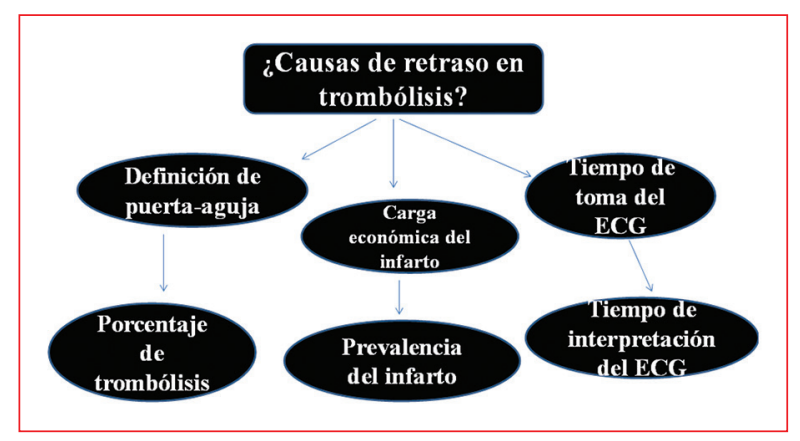

Figura 1. Ejemplo de mapa mental.

importantes de una lectura de un artículo o libro. Deben ser breves, fichas de paráfrasis, es una explicación propia, utilizando nuestros términos o ideas para presentar un material de la fuente original. Existen más tipos de fichas de contenido, de elementos y otras. Deben de tener referencias de acuerdo con el formato de citación que se necesita: Vancouver, APA u otro (Tabla 3) ${ }^{12}$.

En cuanto los trabajos de estudios similares al que pretendemos realizar, se facilitará la lectura y resumen identificando y organizando la información en objetivo principal del estudio, población (niños, adultos, ancianos mujeres, hombres, latinos, europeos, etc.) y resultados de acuerdo con el objetivo ${ }^{9}$ (Tabla 4).

\section{¿Por dónde empezamos?}

Los mapas mentales sirven para organizar la información y armar el esqueleto de la redacción de nuestro marco. El mapa mental es una técnica de estudio conocida amable y práctica, en cuyo centro estará la pregunta de investigación. Estos mapas pueden ir cambiando de acuerdo con el avance de la realización del marco teórico y cada vez estar mejor estructurados y $\operatorname{organizados}^{13}$ (Fig. 1). 
Tabla 4. Organizar la información relevante de un artículo

\begin{tabular}{|l|l|l|l|}
\hline Nombre del estudio y año & Objetivo principal & Población, metodología & Resultados \\
\hline $\begin{array}{l}\text { RENASICA 2016 } \\
\text { (citación Vancouver) }\end{array}$ & $\begin{array}{l}\text { Porcentaje de trombólisis y } \\
\text { causas de retraso }\end{array}$ & $\begin{array}{l}\text { Hospitales de 2 nivel } \\
\text { Estudio descriptivo }\end{array}$ & $\begin{array}{l}60 \% \text { trombólisis } \\
40 \% \text { no se interpretó ECG en los } \\
\text { primeros } 10 \text { minutos }\end{array}$ \\
\hline
\end{tabular}

ECG: electrocardiograma

Otra técnica es ordenar la información por secciones. El marco teórico debe tener (como en un cuento) un inicio (introducción), un desarrollo y una conclusión o final. Ayuda realizar un índice de temas y subtemas ${ }^{9}$ (Tabla 5).

Al empezar a escribir debemos definir las variables principales del estudio, exponer las teorías previamente establecidas, datos epidemiológicos y carga económica de la enfermedad (de ser el caso), así como estudios similares al que pretendemos realizar ${ }^{9}$.

Por poner un ejemplo, «Tiempo puerta-aguja en trombólisis en el infarto de miocardio». Iniciaríamos definiendo tiempo puerta-aguja y trombólisis, posteriormente procederíamos a explicar según las teorías ya establecidas por qué el tiempo es fundamental en la trombólisis y por qué es relevante conocer el tiempo de trombólisis cuando no se dispone de cateterismo (tiempo es miocardio), las causas de retraso conocidas, datos epidemiológicos respecto a porcentaje de trombólisis, costo que generan las complicaciones del infarto a las instituciones (magnitud del problema) y, finalmente, estudios similares. EI RENASICA III documentó en los casos de infarto con elevación del ST que el 37.6\% recibió trombólisis y el $15 \%$ angioplastia primaria, el resto llegó fuera de tiempo ${ }^{14}$. En un hospital de salubridad de Tabasco ingresaron 210 pacientes en el año 2012; la trombólisis se utilizó en el $63 \%$ de los pacientes ${ }^{15}$, en cuanto al Instituto Mexicano del Seguro Social (IMSS), la fibrinólisis se realiza en el $40.4 \%$ de los pacientes y la intervención percutánea en un $8.48 \%{ }^{16}$, a diferencia de en un hospital del Instituto de Seguridad y Servicios Sociales de los Trabajadores del Estado (ISSSTE), en el cual en una muestra de 91 pacientes con infarto al miocardio con elevación del intervalo ST (IMEST) recibieron trombólisis el $63 \%$ (57) y angioplastia el 37\% (34) ${ }^{17}$.

\section{Objetivos del marco teórico final}

- Explicar qué motivo la pregunta de investigación.

- Resumir el estado actual del tema: ¿qué se sabe?

- Plantear la necesidad de la investigación (desconocimiento, carga económica, etc.).
Tabla 5. Ejemplo de marco teórico

\begin{tabular}{|l|l|}
\hline $\begin{array}{l}\text { Marco teórico (ejemplo) tiempo puerta-aguja en infarto de } \\
\text { miocardio }\end{array}$ \\
\hline 1 & Definición de tiempo puerta-aguja y trombólisis \\
\hline 2 & Mecanismo de acción de trombólisis y tasa de éxito \\
\hline 3 & Datos epidemiológicos, infartos/año, complicaciones \\
\hline 4 & $\begin{array}{l}\text { Costo de las enfermedades cardiacas, infarto y } \\
\text { complicaciones (carga económica) }\end{array}$ \\
\hline 5 & $\begin{array}{l}\text { Porcentaje de trombólisis y causas de retraso en } \\
\text { estudios similares }\end{array}$ \\
\hline 6 & Estudios en México (SSA, IMSS, ISSSTE) \\
\hline 7 & Estudios en Latinoamérica \\
\hline 8 & Estudios en Estados Unidos y Europa \\
\hline
\end{tabular}

SS: Secretaria de Salud; IMSS: Instituto Mexicano del Seguro Social; ISSSTE: Instituto de Seguridad y Servicios Sociales de los Trabajadores del Estado.

- Comprender la metodología utilizada y los resultados.

- Servir con fuente para comparar los resultados de estudio similares.

- Entender la aportación del estudio al conocimiento del área disciplinaria.

- Sugerir nuevas preguntas de investigación².

\section{Evite el plagio}

No olvide que al redactar no se trascribe el párrafo de un artículo, libro o cualquier fuente a menos que esté entrecomillado, de lo contrario es plagio: Se llama plagiar a usar palabras, presentar una idea o producto de otra persona como si fueran propios, sin acreditar de dónde proviene la información; un ejemplo es copiar y pegar fragmentos o párrafos de un trabajo en el marco «nuevo», algo muy común en quien no sabe que eso es fraude, y aunque no es intencional constituye una falta. Tenemos la libertad de sintetizar, utilizar ideas (parafraseo), frases y palabras (entre comillas) de los trabajos de otros investigadores para formar nuestro propio marco, pero el material que utilicemos 
debe ser citado y documentar que son ideas y la información del trabajo de otras personas ${ }^{18}$.

\section{Recomendaciones finales}

Los conceptos generales como infarto, sepsis, estado hiperosmolar, etc., se escriben tal cual pero se cita la fuente principal. Cuando defina un concepto consulte asociaciones (asociación de anestesiólogos), consensos (tercer consenso de sepsis), colegios (colegio de cirujanos); no cite el artículo que tiene la definición del concepto (la mayoría toma de referencia la máxima autoridad American Heart Association AHA], American Diabetes Association [ADA], etc.).

No tome datos del marco de estudios originales, solo sus resultados; por ejemplo, la prevalencia de infarto en México es del $12 \%$, el estudio no lo dice en sus resultados, es parte de su marco, lo que debe hacer es consultar el estudio original que habla de la prevalencia y que se usó (citó) para elabora el marco teórico de dicho estudio.

Sí podemos consultar estudios que se utilizaron en el marco teórico de un estudio similar al nuestro, pero siempre consultando el original.

No se olvide del parafraseo y dar el crédito a los autores.

Toda definición debe expresarse en términos afirmativos, no negativos, esto evitará confusión.

Emplee un lenguaje claro, con significado preciso y unitario.

\section{Bibliografía}

1. Hernández-Meléndrez E. Cómo escribir una tesis. Metodología de investigación [Internet]. México: Escuela Nacional de Salud Pública, 2006 [fecha de consulta: 26/09/2019]. Disponible en: http://biblioteca.ucv.cl/ site/servicios/documentos/como_escribir_tesis.pdf
2. Munch $L$, Ángeles E. Métodos y técnicas de investigación. 4. a ed. México: Trillas; 2009.

3. Rivera-García P. Marco teórico, elemento fundamental en el proceso de investigación científica [Internet]. Zaragoza, España: Laboratorio de aplicaciones computacionales, UNAM [fecha de consulta: 25/09/2019]. Disponible en: http://bivir.uacj.mx/Reserva/Documentos/rva200334.pdf

4. Cauas D. Elemento para un proyecto de investigación. Funciones y construcción del marco teórico [Internet]. [fecha de consulta: 20/04/2019]. Disponible en: http://webjam-upload.s3.amazonaws.com/apuntesinvest_ cauas_1539_.pdf

5. Matos-Columbié ZC. La construcción del marco teórico en la investigación. Apuntes para su orientación metodológica en la tesis. EduSol. 2012;10(31):1-14.

6. Vera-Carrasco O. Cómo escribir artículos de revisión. Rev Med La Paz. 2009;15(1):63-9.

7. Villa-Romero A, Moreno-Altamirano L, García de la Torre GS. Epidemiología y estadística en Salud Pública. Mac Graw-Hill; 2011.

8. Turabian KL. Finding useful sources. En: Turabian KL, editora. MLA Handbook for writers of research paper. $7^{\text {th }}$ edition. New York: University of Chicago Press; 2009.

9. Moncada-Hernández SG. Cómo realizar una búsqueda de información eficiente. Foco en estudiantes, profesores e investigadores en el área educativa. Inv Ed Med. 2014;3(10):106-15.

10. Pinillo León AL, Cañedo Andalia R. El MeSH: una herramienta clave para la búsqueda de información en la base de datos Medline. Acimed. 2005;13(2).

11. Hernández-Supiera R, Fernández-Collado $C$, Baptista-Lucio P. Metodología de la investigación. 6. a ed. México: Mc Graw-Hill; 2014.

12. Robledo-Mérida C. Recolección de datos. En: Robledo Mérida C, editor. Técnicas y proceso de investigación. Guatemala: Talleres de litografía de Mercagraf; 2003. pp. 63-73.

13. Roig-Zamora J, Araya-Ramírez J. El uso del mapa mental como herramienta didáctica en los procesos de investigación. Revista e-Ciencias de la Información. 2013;3(2):1-22.

14. Martínez-Sánchez C, Borrayo G, Carrillo J, Juárez U, Quintanilla J, Jerjes-Sánchez C; RENASICA III Investigators. Abordaje clínico y evolución hospitalaria en pacientes mexicanos con sindrome coronario agudo: El Tercer Registro Nacional de Síndromes Coronarios Agudos (RENASICA III). Arch Cardiol Méx. 2016;86(3):221-32.

15. Baños-González MA, Henne-Otero OL, Torres-Hernández ME, Torres-López JE, González-Aguilar CL, Sangeado-Santos M, et al. Factores asociados con retraso en la terapia de reperfusión en infarto agudo de miocardio con elevación del segmento ST (IMCEST) en un hospital del sureste mexicano. Gac Med Méx. 2016;152:495-502.

16. Borrayo-Sánchez G, Madrid-Miller A, Arriaga-Nava R, Ramos-Corrales MA, García-Aguilar J, Almeida-Gutiérrez E. Riesgo estratificado de los síndromes coronarios agudos. Resultados del primer RENASCA-IMSS. Rev Med Inst Méx Seguro Soc. 2010;48(3):259-64.

17. Raymundo ACA, Navarrete AH, Terrazas LVM. Mortalidad postrombólisis versus angioplastia en pacientes con síndrome isquémico coronario agudo, ingresados en la Unidad de Cuidados Intensivos del Hospital ISSSTE Zaragoza. Evid Med Invest Salud. 2012;5(2):52-8.

18. Turabian KL. Plagiarism and acadmic integrity. En: Turabian KL. Handbook of writers of research paper. $7^{\text {th }}$ ed. New York: University of Chicago Press; 2009. 